\title{
Validation of the Automatic Computation of the Ejection Fraction from Cine-MRI
}

\author{
A. Pednekar ${ }^{1}$, I.A. Kakadiaris ${ }^{1}$, U. Kurkure ${ }^{1}$, R. Muthupillai ${ }^{2}$, and S. Flamm ${ }^{3}$ \\ 1 Visual Computing Lab, Dept. of CS, Univ. of Houston, Houston, TX 77204, USA \\ ioannisk@uh.edu \\ 2 Philips Medical Systems North America, Bothell, WA, USA \\ 3 Dept. of Radiology, St. Luke's Episcopal Hospital, Houston, TX 77030, USA
}

\begin{abstract}
In this study of fourteen volunteers, we evaluated our automatic left ventricular (LV) segmentation algorithm w.r.t. ejection fraction (EF) computation using short-axis cardiac cine MR data.
\end{abstract}

\section{Introduction}

Experts routinely perform estimation of cardiac EF manually by tracing the boundaries of the myocardium in short-axis cine MR (CMR) images. An automatic segmentation method would eliminate intra- and inter-observer variability by providing accurate and consistent results. The automation of the LV segmentation includes challenges related to the automatic localization of the LV in CMR scans, the inherently fuzzy nature of the CMR scan due to heart dynamics, and the presence of the papillary muscles inside the LV cavity. Our automatic LV myocardial surface extraction method automatically localizes the LV using region dynamics maps and integrates a fuzzy affinity-based region segmentation approach with the elastically adaptive physics-based deformable models [1]. The goal of this paper is to present our investigation on the clinical feasibility of our automatic LV segmentation method for EF computation.

\section{Method}

Contiguous $10 \mathrm{~mm}$ short-axis slices were imaged on a $1.5 \mathrm{~T}$ commercial imager (Philips Gyroscan NT-Intera) to cover the entire LV using a cine bFFE sequence (TE/TR/flip: 3.2/1.6/55 deg; 38-40 msec temporal resolution) with VCG gating, in eight $(6 \mathrm{~m} / 2 \mathrm{f})$ asymptomatic volunteers and $\operatorname{six}(3 \mathrm{~m} / 3 \mathrm{f})$ symptomatic subjects. Two observers outlined the endocardial contours on each slice of the enddiastolic and end-systolic phase using EasyVision 5.0 (Philips Medical Systems). A separate post-processing workstation was used to compute the EF using our algorithm.

\section{Results}

The agreement between the manual and automatic method was assessed using the Bland and Altman's method. The EF from the manual tracings is computed 
by excluding the extreme basal slices, which contain both ventricular and atrial myocardium and hence are traced up to the junctions of the atrium and the ventricle. The delineation of the endocardial boundary in the extreme apical slice presents difficulties due to the large partial voluming effect of dense trabeculae carneae (especially in the end-systolic phase), in addition to intensity coil falloff. Thus, we exclude the extreme apical slice from the EF computation.

The EF computed using the two methods is in good agreement: Reader 1: mean bias EF (in \%): 3.1325 +/- 4.7655; limits of agreement (+/- 2SD): +12.6635 to -6.3985 . Reader 2: mean bias EF (in \%): $0.0102+/-3.8772$; limits of agreement (+/- 2SD): +7.7645 to -7.7442 . Inter-observer variability between the two readers is as follows: mean bias EF (in \%): $-3.1223+/-2.9653$; limits of agreement (+/- 2SD): +2.8082 to -9.0528 (Fig. 21).

\section{Discussion and Conclusion}

Although bFFE images provide high intrinsic myocardial to blood pool contrast, the trabeculae carneae near the apex and the dorsal wall, and the two principal papillary muscles near the sternocostal wall and the diaphragmatic wall (projecting out of the myocardial wall) pose challenges to the automatic extraction of endocardial contours. Our method overcomes the problem posed by the spatial discontinuity of the intensity gradient between the myocardium and the blood due to the papillary muscles and accurately segments the mid-ventricular slices. Fig. 1 depicts the strength of our algorithm in overcoming the effect of the papillary muscles. However, our method could not extract endocardial contours at the extreme apical and basal slice. Our preliminary results indicate that our automatic algorithm estimates the EF reliably, and the bias and the limits of agreement are comparable to inter-observer variability inherent in manual methods. Further comprehensive clinical validations using data from thirty cases is currently underway.
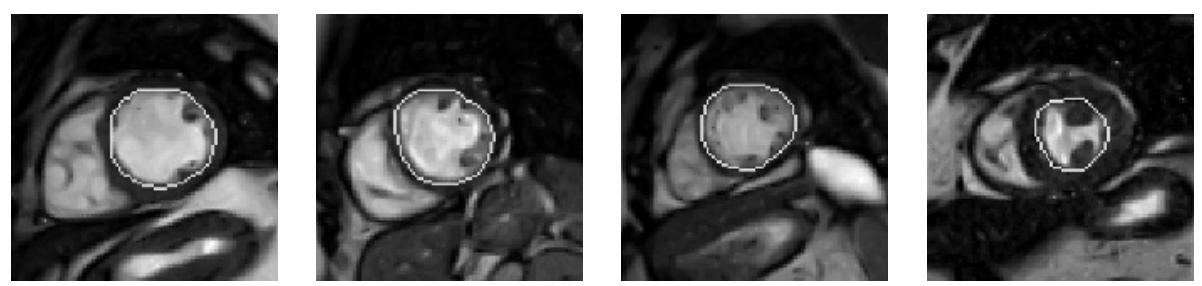

Fig. 1. Extracted endocardial boundaries for the (a) $6^{\text {th }}$ slice of the end-diastolic phase (Subject-6), (b) $5^{\text {th }}$ slice of the end-diastolic phase (Subject-14), (c) $5^{\text {th }}$ slice of the end-diastolic phase (Subject-7), and (d) $7^{\text {th }}$ slice of the end-diastolic phase (Subject11). 

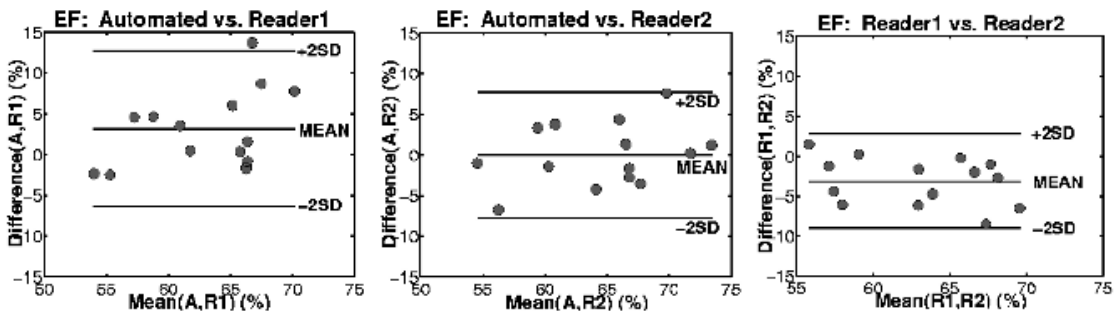

Fig. 2. Bland-Altman Plots for the EF computation from fourteen subjects.

\section{References}

1. A. Pednekar, I. Kakadiaris, R. Muthupillai, and S. D. Flamm. Intensity and morphology-based energy minimization for the automatic segmentation of the myocardium. To appear in the IEEE Workshop on Variational and Level Set Methods (VLSM'03), Nice, France, October 11-12 2003. 